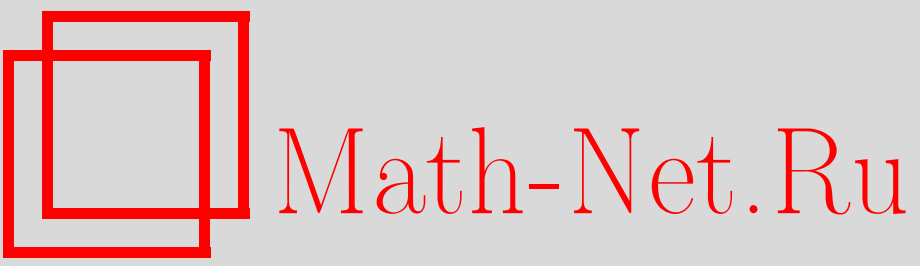

М. Л. Бланк, Спектральные свойства левых треугольных матриц, УМН, 2001, том 56, выпуск 1, 155-156

DOI: https://doi.org/10.4213/rm361

Использование Общероссийского математического портала Math-Net.Ru подразумевает, что вы прочитали и согласны с пользовательским соглашением

http://www.mathnet.ru/rus/agreement

Параметры загрузки:

IP : 18.234 .197 .8

26 апреля 2023 г., 13:50:35 


\title{
СПЕКТРАЛЬНЫЕ СВОЙСТВА ЛЕВЫХ ТРЕУГОЛЬНЫХ МАТРИЦ
}

\author{
М. Л. БЛАнК
}

Для семейства левых треугольных $(n \times n)$-матриц с двоичньми $(0,1)$ элементами получено асимптотически точное (при $n \rightarrow \infty$ ) представление для полной системы собственных векторов и собственных значений. В частности, показан асимптотически линейный рост каждого собственного значения по $n$. Аналогичный резултат получен также для более общего (со специальным образом шкалируемьми элементами) семейства левых треугольных матриц. Эти результаты применены для анализа эргодических свойств одного семейства хаотических отображений.

1. Левой треугольнй (по аналогии с верхней треугольной) $(n \times n)$-матрицей с двоичными $(0,1)$ элементами назовем матрицу $A_{n}=\left(a_{i j}\right)$, элементы которой вплоть до побочной диагонали равны единице, а все остальные равны нулю, т.е. $a_{i j}=1$ при $1 \leqslant i \leqslant n-j+1$. Несмотря на вполне классический вид, спектральные свойства подобных матриц в литературе не описаны, возможно, ввиду отсутствия при конечном размере матрицы $n$ явного представления для ееспектра. Обозначим через $\lambda_{1}^{(n)}, \lambda_{2}^{(n)}, \ldots, \lambda_{n}^{(n)}$ собственные значения матрицы $A_{n}$, упорядоченные по убыванию их абсолютных величин, и через $\left\{e_{k}^{(n)}\right\}_{k=1}^{n}-$ соответствующий набор ортогональных собственных векторов. Как обычно, через $(e)_{i}$ мы обозначаем $i$-й элемент вектора $e \in \mathbb{R}^{n}$, а через $\|e\|$ - его $\mathbf{L}^{2}$-норму.

Теорема 1.1. Пусть $A_{n}$ - левая треугольная матрица. Тогда при каждом $k \in\{1, \ldots, n\}$ выполнено

$$
\lambda_{k}^{(n)}=(-1)^{k+1} \frac{n}{(k-1 / 2) \pi}+O(1), \quad\left(e_{k}^{(n)}\right)_{i}=\cos \left(\frac{(k-1 / 2)(i-1)}{n} \pi\right)+O\left(\frac{1}{n}\right) .
$$

ДоКАЗАТЕЛЬСтвО этого результата основывается на следующих утверждениях. Заметим, что $\left(A_{n} v\right)_{i}=\sum_{j=1}^{n-i+1} v_{j}$ для любого вектора $v \in \mathbb{R}^{n}$.

Лемма 1.1. Для любой $C^{1}$-гладкой функции $f:[0,1] \rightarrow[-1,1]$ с производной, не превышающей 1 по модулю, выполняется

$$
\sum_{j=1}^{n-i+1} f\left(\frac{j-1}{n}\right)=n \sum_{j=1}^{n-i+1} f\left(\frac{j-1}{n}\right) \cdot \frac{1}{n}=n \int_{0}^{1-\frac{i-1}{n}} f(s) d s+O(1) .
$$

ЛЕмма 1.2. Oператор $L: f(x) \rightarrow \int_{0}^{1-x} f(s) d s$ в $\mathbf{L}^{2}$ имеет полную систему ортогональных собственных функиий $E_{k}(x):=\cos ((k-1 / 2) \pi x)$ с собственными значениями $\mu_{k}:=(-1)^{k+1}[(k-1 / 2) \pi]^{-1} \partial \Omega_{s} k=1,2, \ldots$.

Лемма 1.3. Пусть равенство $A v=\mu v+\xi$ выполнено для симметричной матрицы $A$, некоторых векторов $v, \xi \in \mathbb{R}^{n} c\|\xi\| \leqslant \varepsilon\|v\|$ и числа $\mu$. Тогда ближайшее $к \mu$ собственное значение $\lambda$ матрицы $A$ удовлетворяет неравенству $|\lambda-\mu| \leqslant \varepsilon$, и если его кратность равна 1 , то для соответствующего собственного вектора е выполнено $\|e-v\| \leqslant O(\varepsilon) \cdot\|v\|$.

Идея доказательства здесь состоит в том, что $\left(A-\xi v^{*} /\|v\|^{2}\right) v=\mu v$ (см. также [1]).

2. Изучим теперь более общий класс левых треугольных матриц. Пусть $\phi:[0,1] \rightarrow(0,1]$ $-C^{1}$-гладкая функция. Функция $\phi$ порождает семейство левых треугольных $(n \times n)$-матриц: $\left(A_{n}^{\phi}\right)_{i, j}:=\phi((j-i+1) / n)$ при $i \leqslant j$ и равно 0 в противном случае. Отметим, что двоичная левая треугольная матрица удовлетворяет этому условию при $\phi \equiv 1$.

Используя, как и в предыдущем разделе, то свойство, что основной вклад в “форму" собственных векторов дают собственные функции интегрального оператора $L_{\phi}: f(x) \rightarrow \int_{0}^{1-x} \phi(s) f(s) d s$, можно доказать асимптотически линейньй по $n$ рост собственных значений матрицы $A_{n}^{\phi}$.

Работа выполнена при частичной поддержке Российского фонда фундаментальных исследований, грантов INTAS и CRDF. 
Tеорема 2.1. Пусть $\mu$ и $f_{\mu}-$ собственное значение и собственная функция оператоpа $L_{\phi}$. Тогда $\forall n$ найдутся собственное значение $\lambda=n \mu+O(1)$ и собственный вектор $e \in \mathbb{R}^{n}$ матрицы $A_{n}^{\phi}$ такой, что $(e)_{i}=f_{\mu}((i-1) / n)+O(1 / n)$ при всех $i=1,2, \ldots, n$.

Предположим, что функция $\phi$ является невозрастающей, и обозначим через $\mathbf{S}^{n} \subset \mathbb{R}^{n}$ множество положительных векторов $v$, зависимость элементов которых от их номера является монотонно убывающей и вогнутой функцией. Введем также нелинейный оператор $B_{n}: \mathbb{R}^{n} \rightarrow \mathbb{R}^{n} \cup(\infty)^{n}$, определяемшій соотношением $\left(B_{n} v\right)_{i}:=\left(A_{n}^{\phi} v\right)_{i} /\left(A_{n}^{\phi} v\right)_{1}$.

ЛЕмма 2.1. Множество $\mathbf{S}^{n}$ является $B_{n}$-инвариантным, и для любого вектора $v \in \mathbf{S}^{n}$ последовательность $\left\{B_{n}^{m} v\right\}_{m}$ сходится при $m \rightarrow \infty$ в $\mathbf{L}^{2}$-норме $к$ ведущему собственному вектору $e_{1}^{(n)}$ матрицы $A_{n}^{\phi}$.

В качестве следствия, выбирая векторы $v \in \mathbf{S}^{n}$, соответствующие минимальному и максималшному $\left(A_{n}^{\phi} v\right)_{1}$ (а именно, $(v)_{i}=(n-i+1) / n$ и $\left.(v)_{i} \equiv 1\right)$, мы немедленно получаем, что

$$
\frac{n}{2} \int_{0}^{1}(1-s) \phi(s) d s+O(1) \leqslant \lambda_{1}^{(n)} \leqslant n \int_{0}^{1} \phi(s) d s+O(1) .
$$

Подобным путем (но для несколшко более сложного множества $\mathbf{S}^{n}$ ) можно получить также оценки и для остальных элементов спектра.

3. Применим теперь полученные резултаты для анализа эргодических свойств одного семейства хаотических отображений. Необходимые определения и утверждения, связанные с хаотическими отображениями, можно найти, например, в [2].

Для фиксированного натурального $n$ рассмотрим кусочно линейное отображение $T_{n}:[0,1] \rightarrow$ $[0,1]$, определяемое как $\left.T_{n}\right|_{\left[\frac{i-1}{n}, \frac{i}{n}\right)}(x):=\frac{i}{n} x$ для $i \in\{1,2, \ldots, n\}$. Это отображение является топологически перемешивающим, и его вторая итерация является кусочно растягивающим отображением. Поэтому имеется единственная абсолютно непрерьвная $T_{n}$-инвариантная мера (см. [2]).

Лемма 3.1. Топологическая әнтропия отображения $T_{n}$ удовлетворяет соотношению $h_{\mathrm{top}}\left(T_{n}\right)=\ln \left(\frac{2 n}{\pi}+O(1)\right)$.

НАБРОСОК дОКАЗАТЕЛЬСТВА. Заметим, что отображение $T_{n}$ является марковским по отношению к разбиению на интервалы монотонности. Поэтому соответствующая символическая динамика определяется оператором сдвига в пространстве последовательностей из $n$ символов, удовлетворяющих условию, что за $k$-м символом может следовать любой из первых $(n-k+1)$ символов. Таким образом, соответствующая матрица переходов является левой треугольной. Теперь требуемое неравенство следует из теоремы 1.1, поскольку топологическая энтропия как марковского отображения, так и соответствующего оператора сдвига равна логарифму наибольшего (первого) собственного значения матрицы перехода.

\section{СПИСОК ЛИТЕРАТУРЫ}

[1] B. N. Parlett. The Symmetric Eigenvalue Problem. Englewood Cliffs, NJ: Prentice-Hall, 1980. [2] M.L. Blank. Discreteness and Continuity in Problems of Chaotic Dynamics. Providence, RI: Amer. Math. Soc., 1997. 